\title{
La Forma Espacial de la Isla de Calor en la Ciudad de Guayaquil
}

\section{The Spatial Form of the Urban Heat Island in Guayaquil}

Carlos Palacios Portés ${ }^{a}$, Vicente Francisco González Burneo ${ }^{b}$, Santiago Raymundo Dick Zambrano ${ }^{c}$, Melissa Gianella Coello Jairala ${ }^{d}$

INFORMACIÓN DEL ARTÍCULO

Fecha de recepción:10 de febrero del 2017

Fecha de aceptación: 22 de marzo del 2017

${ }^{a}$ Magíster en impactos ambientales Diploma superior en pedagogía universitaria. Universidad de Guayaquil. Facultad de Arquitectura y Urbanismo. Universidad Espíritu Santo - Ecuador, Facultad de Arquitectura e Ingeniería Civil. E-mail: palaciospc@ug.edu.ec,

$b$ Magíster en administración de empresas, candidato a Doctor en Ciencias. Universidad de Guayaquil. Facultad de Arquitectura y Urbanismo.

E-mail: vicente.gonzalesb@ug.edu.ec

c Magíster en tecnologías de la construcción de viviendas a bajo costo. Universidad de Guayaquil. Facultad de Arquitectura y Urbanismo.

E-mail: santiago.dickz@ug.edu.ec

$\bar{d}$ Arquitecta, Universidad de Guayaquil.

E-mail: melissa.gianella@hotmail.com

\begin{abstract}
Resumen
Uno de los impactos ambientales de importancia macro-escalar que le conciernen a la ciudad de Guayaquil es el de la presencia del fenómeno "Isla de calor", como consecuencia de la configuración geométrica de su casco empresarial que marca un pico de $+5,32^{\circ} \mathrm{C}$ con respecto a los registros meteorológicos locales y que ha llegado a comprometer la calidad del aire a nivel del suelo y los patrones de transferencia térmica con el que se encuentra inmediatamente sobre la cubierta de los edificios. El presente estudio confirma la existencia del fenómeno con evidencia empírica y pretende ilustrar su aspecto espacial basado en la medición de variables geométricas que denotan una elevada intensidad de usos de suelo en calles demasiado angostas en relación a la altura de las edificaciones que las flanquean.
\end{abstract}

Palabras Clave

Isla urbana de calor, calor antropogénico, geometría urbana, factor de cielo visible, cañón urbano, intensidad de uso de suelo.

Clasificación JEL: O18, P25.

\begin{abstract}
One of the macro-scale environmental impacts that concern the city of Guayaquil is the existence of an "Urban Heat Island" phenomenon. This is a consequence of the geometric configuration of Guayaquil's business center. It has a $+5,32^{\circ} \mathrm{C}$ peak over the local weather registries. It affects the ground level air quality. It also affects the thermal transference patterns with the layer over the buildings roofs. This work confirms the phenomenon's presence with empiric evidence. It aims to illustrate the spatial aspect based on the measure of geometric variables denoting a high land use in narrow streets regarding the height of the buildings on both sides.

Keywords

Urban heat island, anthropogenic heat, urban geometry, sky view factor, urban canyon, land uses.
\end{abstract}

JEL Classification: 018, P25. 


\section{Introducción}

Todos los organismos vivos modifican en alguna medida su hábitat en busca de protección y alimento, pero es sin lugar a dudas el animal humano el que ha adaptado el medio natural a sus necesidades espaciales para crear ecosistemas urbanos hechos a su medida. Una de las consecuencias de esta intervención es la formación de la "isla urbana de calor" que, en términos sencillos, es un fenómeno que ocasiona la máxima diferencia de temperaturas nocturnas de una zona urbana con respecto al entorno rural inmediato de la ciudad. Los causales de la isla urbana de calor dependen de los tipos de cobertura de las superficies expuestas al sol, los flujos radiantes de energía derivados de los usos de suelo, la densidad poblacional, la masa térmica de los cuerpos y la forma espacial que éstos definen en el contexto; esto deja entrever que, más que asuntos exclusivos de meteorología o física del calor, tales variables trascienden a la planificación espacial de expansión y ordenamiento territorial, y también de corrección de asentamientos poblacionales informales.

Lo que las ciudades con sectores urbanos heterogéneos tienen en común es que sus cascos empresariales retienen la ganancia térmica solar y el calor antropogénico por más tiempo que los demás no sólo por su elevada inercia térmica, sino también un perfil urbano definido por la altura de los edificios (Takkanon, 2016) que confina una capa atmosférica conocida como "palio urbano" (Moreno García, 1997). Por otro lado, la alta intensidad de usos y ocupa- ción de suelo ${ }^{1}$ hacen que las calles se comporten como conductos longitudinales denominados "cañones urbanos" que dificultan el intercambio térmico del aire del palio urbano con un sumidero de calor de mayores proporciones, mientras mayor sea la altura de los edificios en relación al ancho de vía.

Los estudios sobre islas de calor son abundantes para ciudades emplazadas en latitudes de la zona templada, pero no hay muchos para la faja intertropical (Rajagopalan, Chuan Lim, \& Jamei, 2014); a propósito de esto, existen ciudades como Muar, Malasia que, además de poseer un clima similar al de Guayaquil, no tuvieron una planificación urbana frente a la rápida ocupación del suelo y ocasionaron cañones urbanos profundos que obstruyen el paso del viento (Rajagopalan, Chuan Lim, \& Jamei, 2014) con islas de calor de hasta $3,2^{\circ} \mathrm{C}$. En cambio, también se han reportado casos como el de Chennai, India, en los que también hay cañones urbanos pronunciados, que canalizan la ventilación de mejor manera que en las áreas con cielo despejado y mantienen el confort térmico debido a que el trazado urbano que favorece la ventilación. En general el viento tiene potencial de mitigar los efectos de la isla urbana de calor, al menos la sensación térmica, siempre que las condiciones geométricas de los trazados urbanos posibiliten su circulación eficiente (Chirag \& Ramachandraiah, 2011).

\footnotetext{
Según indica la Ordenanza sustitutiva de edificaciones y construcciones del cantón Guayaquil vigente, los coeficientes de ocupación del suelo para esta zona urbana varían entre 0,70 y 0,80 , mientras que los de utilización del suelo está entre 6,00 y 8,00 veces el área del lote.
} 
Algunas de las condicionantes físicas y espaciales hasta aquí mencionadas se verifican también en el casco comercial-empresarial de la ciudad de Santiago de Guayaquil, Ecuador $\left(2^{\circ} 25^{\prime} \mathrm{S}\right.$; $\left.79^{\circ} 47^{\prime} \mathrm{W}\right)^{2}$, en cuyo caso se presume la existencia del fenómeno a partir de una imagen satelital rasterizada de la localidad y ciertas características geométricas de cañón urbano que coinciden con los patrones identificados en otras ciudades del mundo, ver figura1. El presente estudio describe el impacto térmico que surten las aglomeraciones edilicias de un entorno completamente intervenido a causa de su forma urbana, para lo cual se ha considerado variables geométricas en lugar de las propiedades calóricas de la masa térmica existente y sus coberturas.

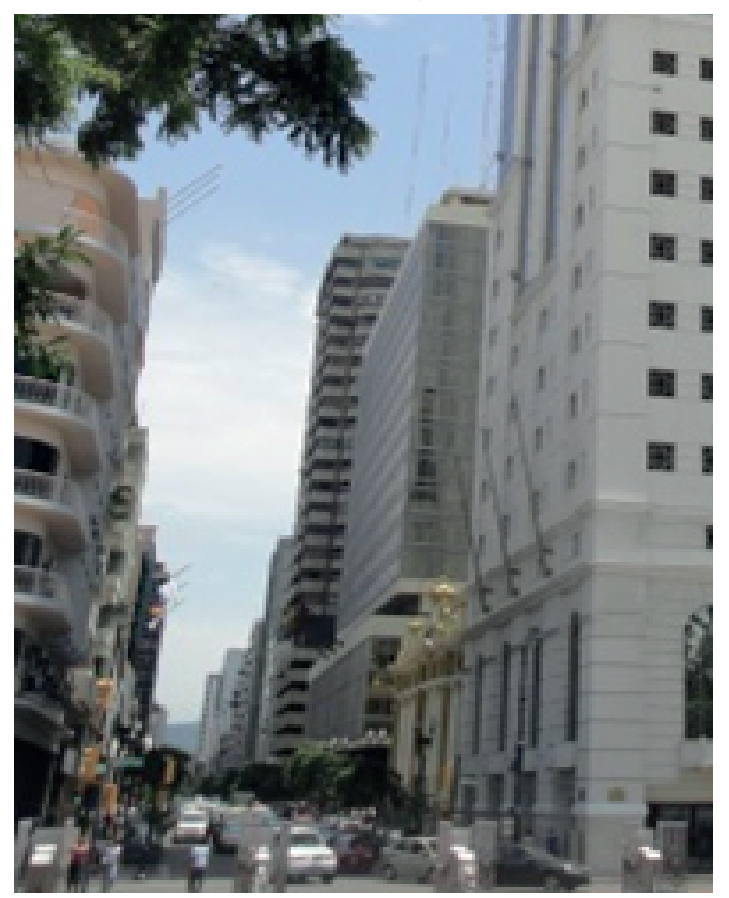

Figura 1. Cañón urbano del Boulevard 9 de octubre en el casco empresarial-comercial de la ciudad de Guayaquil.

\section{Materiales y Métodos}

Para ilustrar la existencia del fenómeno de la Isla Urbana de Calor en la ciudad de Guayaquil se parte de una captura satelital rasterizada del casco comercial y sus alrededores (figura 2), la mancha urbana delata una intensificación del uso del suelo hacia el casco comercial por el oscurecimiento del color azul. Otra lectura de esta misma información es que las gamas del azul hacia el negro y del anaranjado hacia el blanco definen, de forma cualitativa, zonas de mayor $y$ menor temperatura respectivamente. Una tercera interpretación indica que los tonos azules oscuros más intensos corresponden a zonas con mayor masa térmica por metro cuadrado como el río Guayas (al este) y el estero Salado (franja sinuosa en negro al oeste), también a la pista de aterrizaje del aeropuerto (noreste) y el centro de la ciudad (círculo rojo en línea segmentada); mientras que los colores que tienden al cyan corresponden a zonas con edificios más pequeños (incluso se llegan a distinguir manzanas y calles), las áreas dispersas en color blanco son superficies cubiertas de césped (estadios y parques varios), y las partes en anaranjado identifican coberturas vegetales del tipo arbóreo (cerro del Carmen, manglar de la isla Santay); la tabla1 sintetiza esta descripción. De cualquier manera, queda claro que la isla de calor se emplaza en el casco comercial de la ciudad por ser la zona urbana con mayor intensidad de uso y ocupación de suelo. 


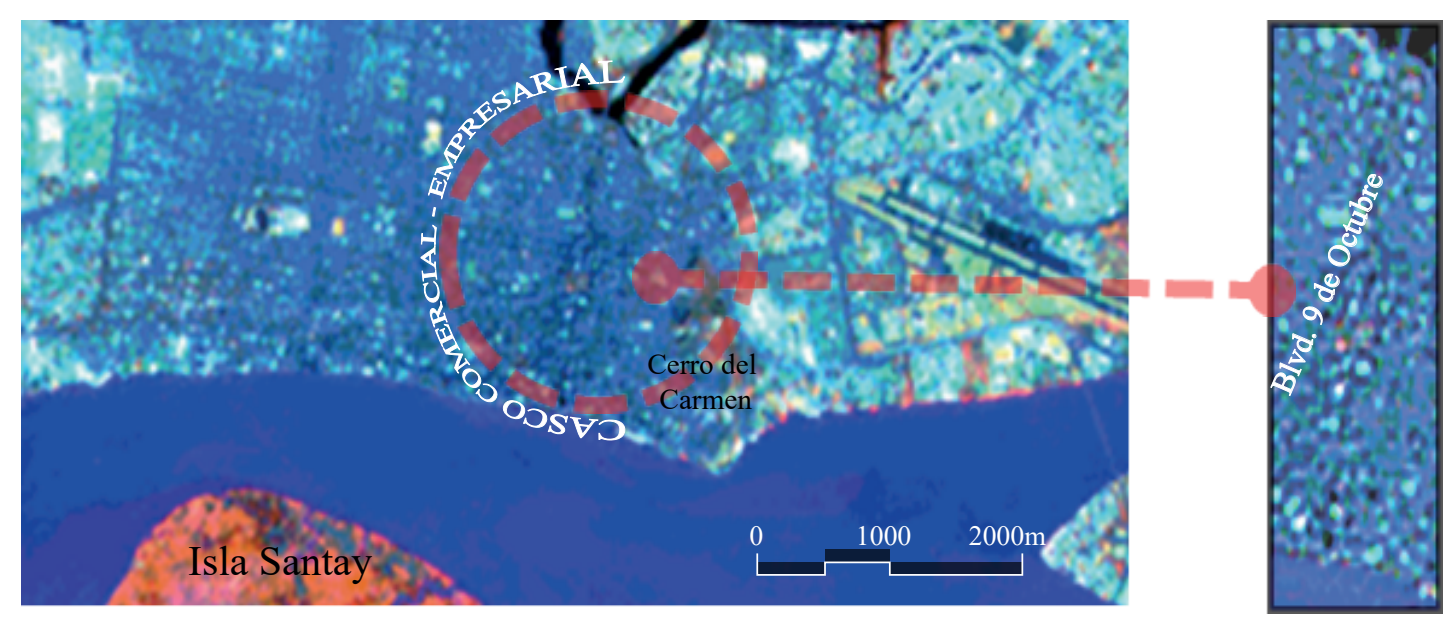

Figura 2. Vista satelital rasterizada del este de la ciudad de Guayaquil ${ }^{3}$ en la que se delimita el casco comercial dentro de un círculo de color rojo. El cuadro de la derecha amplía la vía principal de este sector, el boulevard 9 de Octubre, en el que se aprecia mejor la intensificación de edificaciones en altura por los colores oscuros.

Tabla 1.

Correspondencia cromática de la masa térmica ilustrada en la figura 2.

\begin{tabular}{ll}
\hline Masa térmica & Gradiente cromático \\
\hline Cuerpos de agua & \\
Áreas verdes & \\
Mancha urbana
\end{tabular}

La figura anterior no dispone de una escala de correspondencia color-temperatura, lo que dificulta el dimensionamiento del fenómeno a partirt de ella, sin embargo, basta la sola inspección visual para confirmar que la parte más calurosa del casco empresarial de la ciudad de Guayaquil se desarrolla linealmente a lo largo de su avenida principal (ver la ampliación

${ }^{3}$ La imagen original es propiedad de la M.I. Municipalidad del cantón Guayaquil. de la figura 2). Entonces, se recurrió a realizar un levantamiento de información in situ (Gonçalves · Dos Santos, Gazzola De Lima, \& Sad De Assis) en horas de la noche ${ }^{4}$ durante quince días consecutivos a lo largo de un transecto de 1923,12m de longitud que pasa por las veinte y tres intersecciones viales de la avenida en cuestión; esta labor consistió en recorrer vehicularmente el camino y detenerse en cada intersección vial para tomar la temperatura del aire mediante un termo-higrómetro digital introducido en una caseta meteorológica portátil; el tiempo límite para efectuar el recorrido total fue de treinta minutos con la finalidad de descartar las variaciones de temperatura del aire por el enfriamiento natural que se produce a medida que avanza la noche. La figura 3 muestra el plano del sector con la línea del transecto. 


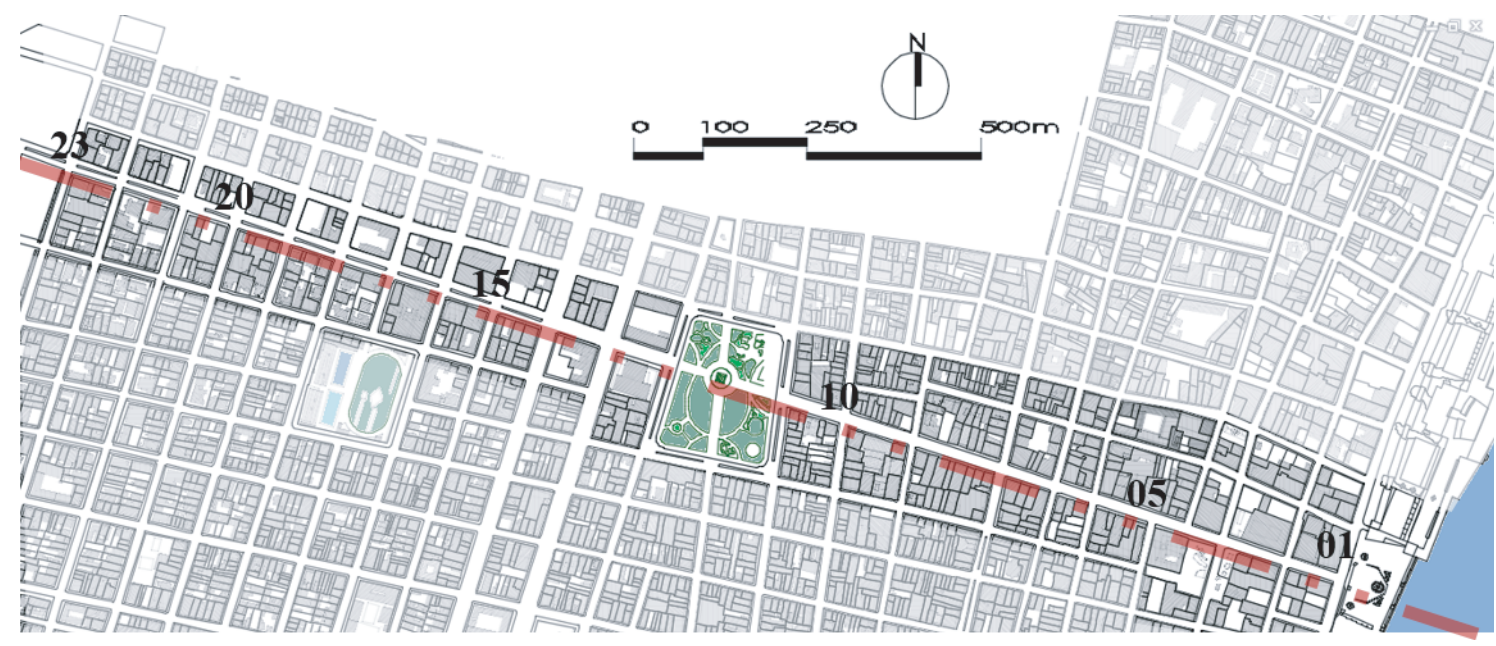

Figura 3. Plano de catastro del centro de la ciudad. La línea segmentada en rojo indica el recorrido aproximado del transecto, y los números sobre ella sugieren el orden de toma de muestras para las intersecciones viales, así el primero es en el malecón Simón Bolívar y el vigésimo tercero corresponde a la avenida Tungurahua.

La aplicación de esta estrategia demandó la precaución de reportar la hora exacta para cada lectura tomada y de evitar distorsiones de la información por ráfagas de viento, lluvia o radiadores de automotores cercanos. Gracias al escaso tránsito vehicular nocturno característico de esta zona fue posible recorrer el trayecto completo al menos dos veces en el tiempo establecido y tomar diez lecturas por parada ${ }^{5}$ de las que más adelante se calculó la media aritmética. Paralelamente se consiguió acceso a los registros horarios de la estación meteorológica fija 842030 SEGU y se procedió a determinar, por interpolación, los valores que corresponden en simultaneidad cronológica a las lecturas de la estación móvil para compararlos por incremento " $\Delta \mathrm{T}$ ", cabe mencionar que no fue posible acceder a la información de ninguna otra estación fija urbana o rural.
Para estudiar las variables geométricas que determinan la resistencia de los cañones urbanos al drenar los flujos radiantes hacia un sumidero de calor se empleó indicadores como el factor de cielo visible "SVF" y la relación altura de edificios-ancho de vía " $\mathrm{h} / \mathrm{w}$ " , ambos con posibilidad de representación gráfica. La forma más común de encontrar el valor de SVF en diferentes puntos de la urbe parte del procesamiento de imágenes fotográficas capturadas por una cámara con lente de ojo de pez, pero se descartó este método fundamentalmente por razones de seguridad social y vial; también se omitió el empleo de herramientas GIS debido a que no se tuvo acceso a información relevante. Lo anterior determinó tomar un camino alternativo a través de la manipulación con software CAD del plano de catastro de la M.I. Municipalidad de Guayaquil.

${ }^{5}$ El termo-higrómetro arroja lecturas cada tres segundos.

${ }^{6}$ SVF y H/W son las siglas en inglés para Sky View Factor y ratio of Height to Width, respectivamente. 
El plano adquirido contenía datos de líneas de construcción, perímetro de plantas altas y cantidad de pisos de cada edificación pero, como no reportaba alturas, fue necesario asumir un factor común entre niveles de piso terminado equivalente a tres metros que, al multiplicarse por la cantidad de pisos de cada caso, daría como resultado alturas totales aproximadas. Más adelante se procedió a modelar el volumen de cada edificio en el programa Autodesk AutoCad por medio de la extrusión simple de cada contorno perimetral, pero se despreció la altura de las veredas y el mobiliario urbano. La figura4 muestra el modelo tridimensional desarrollado para el entorno inmediato del transecto; las superficies en gris claro son los edificios, los cuadriláteros de contorno gris oscuro son las manzanas vecinas, el plano horizontal en negro representa a las calzadas, el plano horizontal en azul, al río Guayas y todas las manchas verdes, a los árboles.

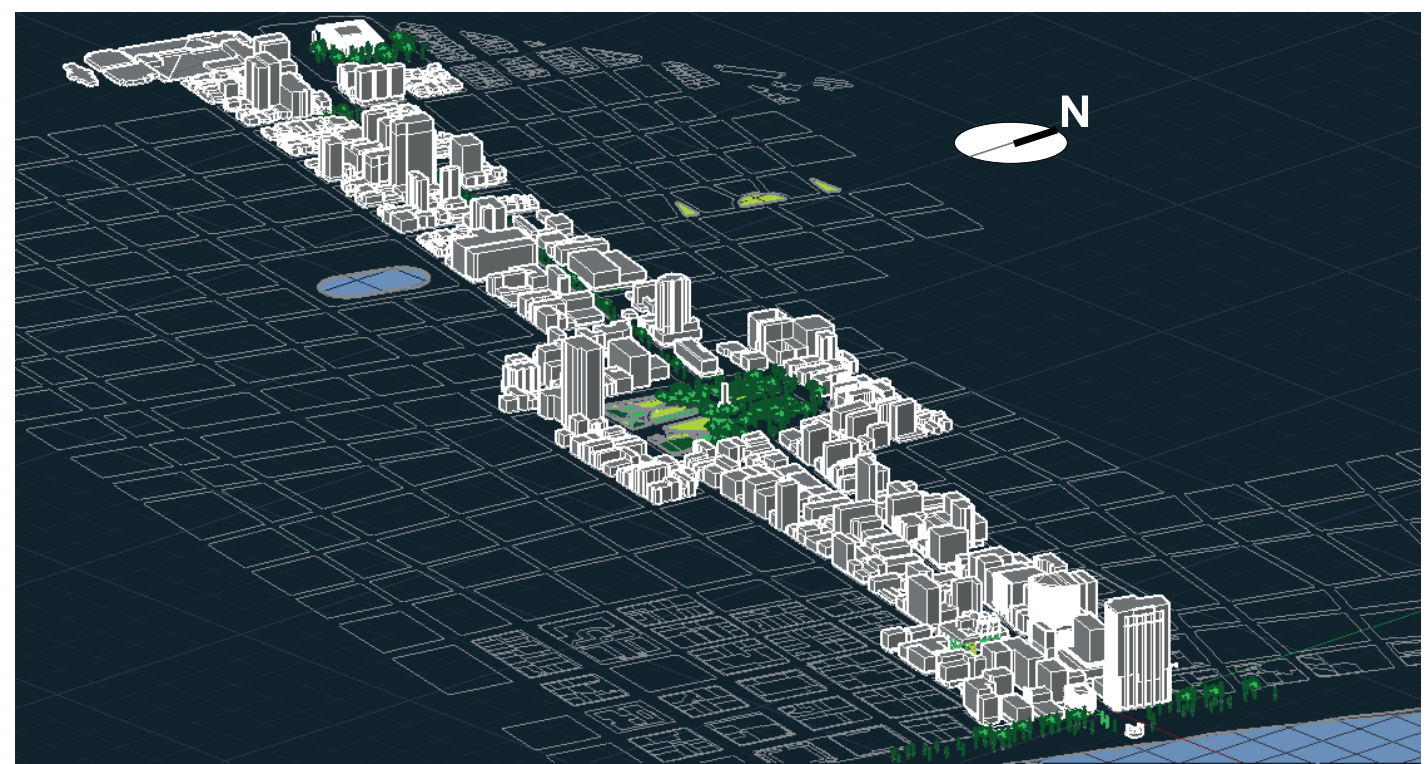

Figura 4. Modelo tridimensional del boulevard 9 de octubre y parte del entorno, a partir de éste se procesó con facilidad los datos necesarios para las variables geométricas.

Es necesario mencionar que, a diferencia de los cuerpos de agua y superficies a nivel del suelo, la vegetación tiene capacidad de modificar el SVF, por tanto debía incluirse en el modelo. La escasa diversidad de especies presentes en el transecto hizo posible la simplificación de la labor, para esto se estandarizó una silueta bidimensional que fue escalada según la altura y diámetro de copa de cada árbol presente en el transecto para después ubicarla en su punto de plantado correspondiente. A efectos de obtener un valor más real de SVF se cuidó que, en vista superior, el plano de cada silueta formara ángulo recto con respecto a la línea imaginaria que une el punto de plantado con el del cruce de los ejes viales.

Una vez completado el modelo tridimensional, el archivo de AutoCad fue exportado a un formato compatible con el 
programa Autodesk Ecotect Analysis para calcular el diagrama solar en cada intersección vial a nivel de la cota del rasante de la calzada, el resultado es una proyección estereográfica del cielo con la silueta de los edificios circundantes que coincide con la representación gráfica de SVF, entonces se guardó esta información en formato de imagen para posteriormente exportarla al programa Matlab SkyViewFactorCalculator y así procesar el valor numérico de SVF.
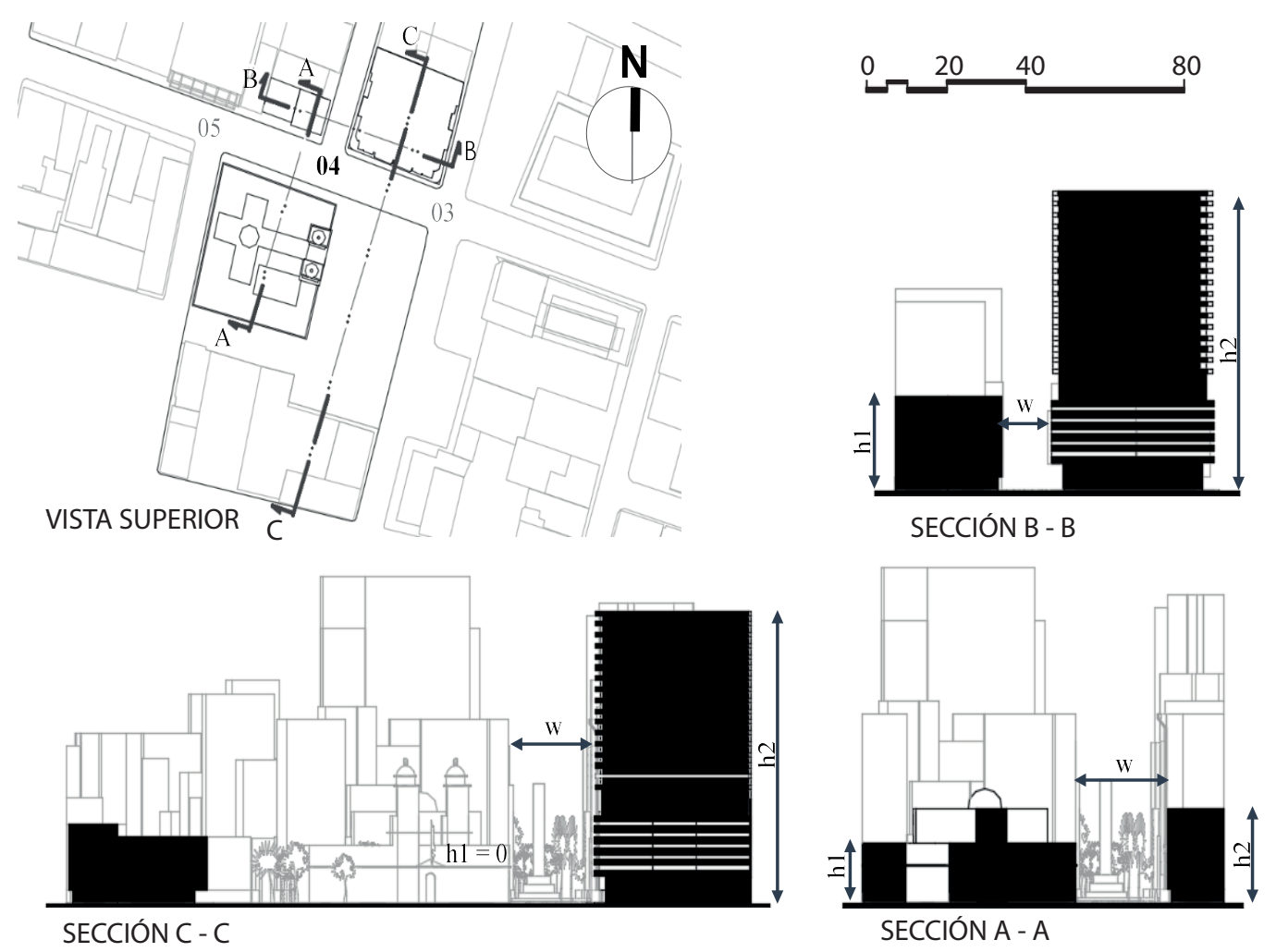

Figura 5. Secciones viales del punto de muestra 04 (Av. General Córdova), aquí se aprecia cómo fueron considerados los valores de "h" y "w".

Una vez concluida la preparación de las variables se procedió a estudiar sus interacciones. Con los valores $\Delta \mathrm{T}, \mathrm{SVF}$ y $\mathrm{h} / \mathrm{w}$ tabulados, primero se identificó el punto con $\Delta \mathrm{T}$ máximo para cuantificar la
Para el cálculo del valor h/w que le asiste a cada punto de muestra se obtuvo el promedio de los valores $\mathrm{h} / \mathrm{w}$ de las secciones viales alrededor de cada intersección vial; las alturas y anchos de vía fueron obtenidos al trazar planos secantes que enlazan perpendicularmente todos los solares esquineros como se aprecia en la vista superior de la figura5.

Cabe indicar que, en todos los casos, los anchos de vía fueron contemplados hasta la línea de soportal. 
modelo numérico. Más adelante se verificó el impacto al confort térmico del transeúnte mediante una carta bioclimática y se empleó el modelo tridimensional para ilustrar bidimensional y estadísticamente la forma del límite de la capa del palio urbano, lo que sirvió para finalmente conocer a qué zona climática local responde el área de estudio dentro de lo que se entiende en el contexto internacional.

\section{Resultados y Discusión}

Después de calibrar el termo-higrómetro digital, la toma móvil de lecturas en sitio confirmó que la variación de temperaturas resulta despreciable $\left( \pm 0,025^{\circ} \mathrm{C}\right)$ siempre que se la realice dentro del lapso de treinta minutos, esto permitió compararlas con seguridad con los registros horarios de la estación fija 842030 SEGU. Se encontró que, aun cuando esta estación fija se encuentra dentro de la ciudad, existe una diferencia positiva a favor de los datos de la estación móvil, lo que confirma que el casco comercial-empresarial de Guayaquil es más caluroso incluso que su entorno urbano inmediato. Está claro que este comportamiento guarda estrecha relación con las características geométricas del sector, por tanto, se presenta la tabla 2 con fines netamente ilustrativos para destacar las variables que intervinieron en el análisis.

Tabla 2.

Ficha técnica de cuatro puntos aleatorios de muestra.

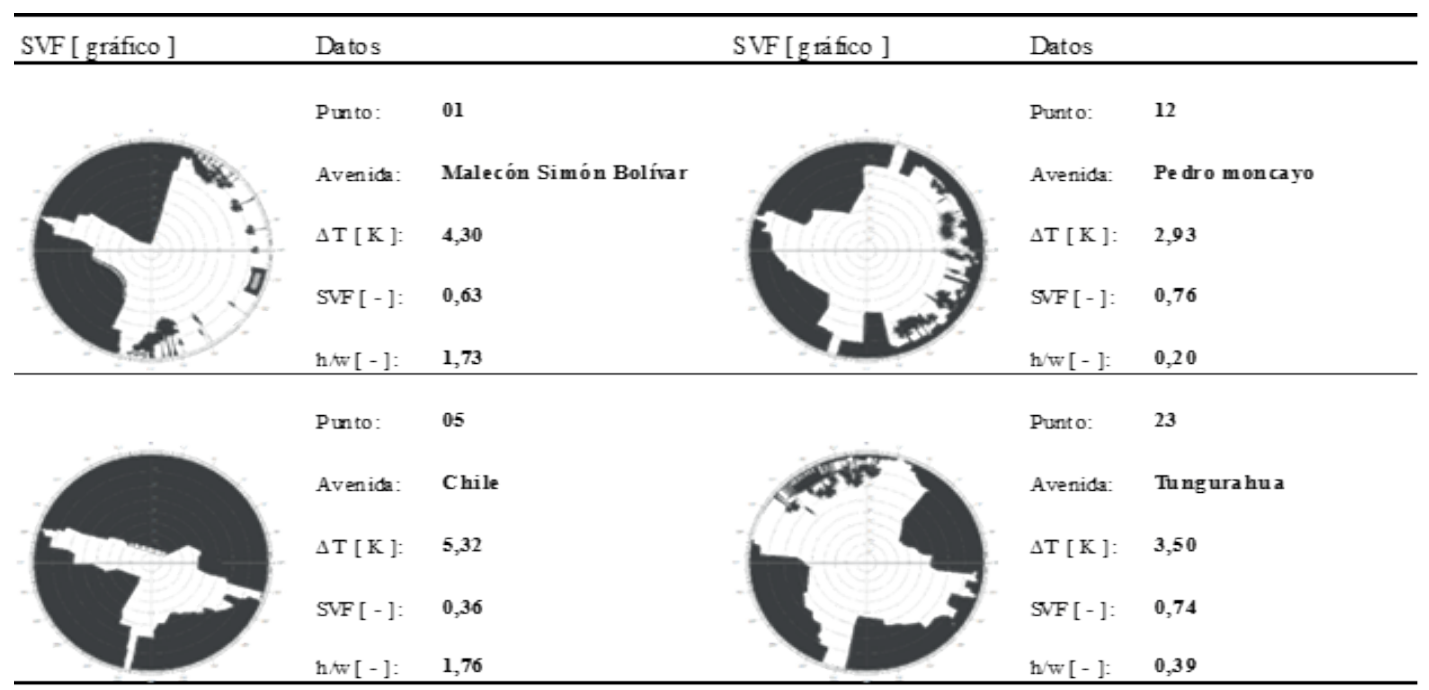

Nota. El color blanco en la representación gráfica de SVF significa la porción de cielo visible, el gris da la idea de los cuerpos que lo obstaculizan. 
La figura 6 grafica todas las variables que fueron tabuladas $\mathrm{y}$ registra la respuesta de todo el transecto. Se ha emplazado las intersecciones viales en el eje de las abscisas según el orden indicado en la figura 3 ; la serie ordenada del lado izquierdo indica los valores $\Delta \mathrm{T}$, y la serie ordenada de la derecha despliega los valores SVF y h/w. Para interpretar la relación de la variable térmica con las variables geométricas es necesario no perder de vista que los valores elevados de $\mathrm{h} / \mathrm{w}$ y SVF significan diferentes cosas: el primero dice en qué sitios predomina la altura de los edificios sobre el ancho vial, el otro marca dónde hay menor obstrucción visual del cielo.

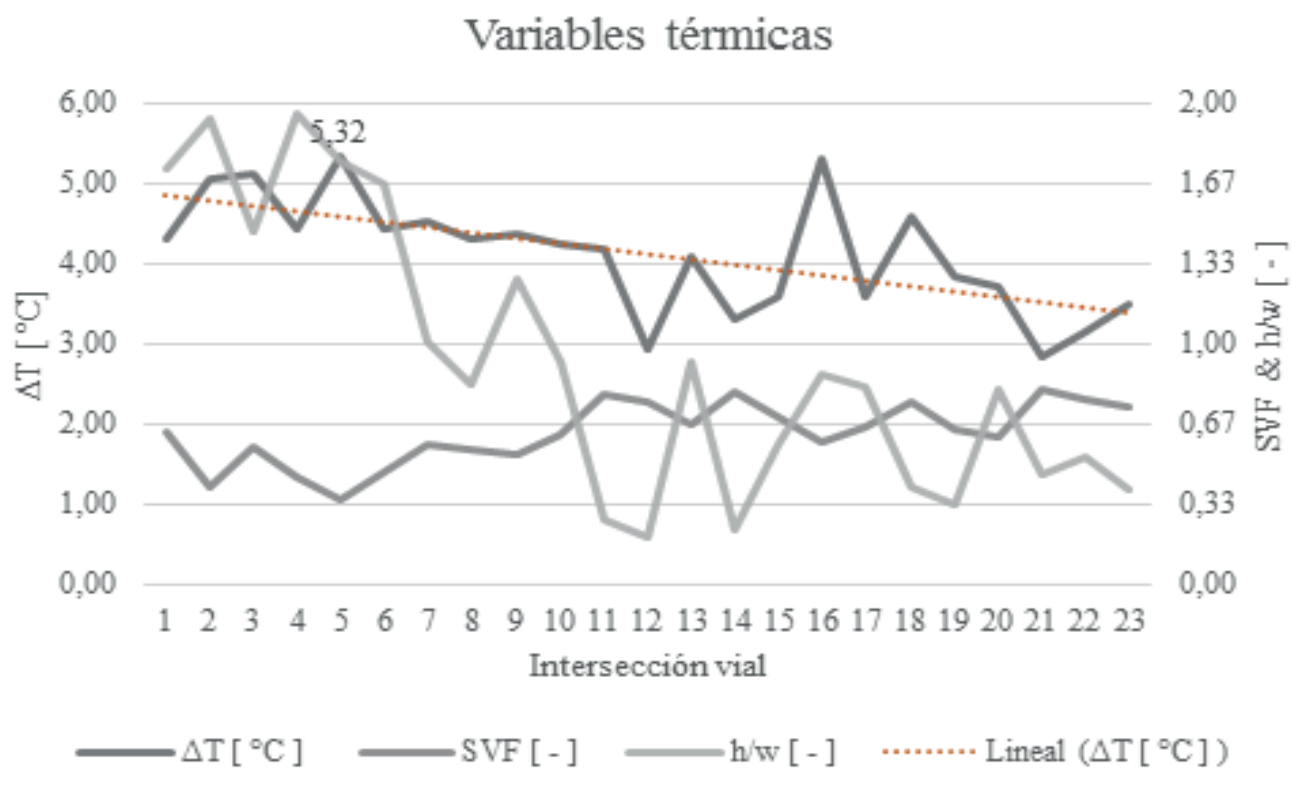

Figura 6. Representación gráfica de las variables $\Delta \mathrm{T}$, SVF y h/w para cada intersección vial del transecto de estudio, la línea segmentada en color anaranjado indica la tendencia al aumento de la temperatura mientras se acerca al punto 01 (donde están los edificios más altos).

En la figura 6 también se aprecia la tendencia al incremento progresivo de temperatura en la medida que disminuye SVF y aumenta $\mathrm{h} / \mathrm{w}$, es decir, se confirma que la temperatura del aire de la capa del palio urbano es mayor en los lugares donde hay concentración de edificios de mayor altura; cabe destacar que se considera que existen "situaciones de cañón"7 cuando el valor de la relación $\mathrm{h} / \mathrm{w}$ es

${ }^{7}$ Se denomina "situaciones de cañón" a las condiciones de proporcionalidad de un cañón urbano que deterioran la calidad del aire a nivel del suelo, debido al incremento de concentración de emisiones, elevadas temperaturas y ventilación que tiende a la disminución. superior a 0,70 (Georgakis \& Santamouris, 2005), en este caso existen catorce puntos que superan ese valor, y el promedio $\mathrm{h} / \mathrm{w}$ de todo el transecto es equivalente a 0,93 , lo que confirma que el Blvd. 9 de octubre presenta situación de cañón. Esta figura además localiza el mayor valor de $\Delta \mathrm{T}$ en el punto 05 con $+5,32^{\circ} \mathrm{C}$, que corresponde a la Av. Chile; según la gráfica, esta intersección presenta el menor valor SVF con 0,36 y el tercer valor más grande de $\mathrm{h} / \mathrm{w}$ a razón de 1,76. Entonces, se constata que la magnitud del 
fenómeno isla urbana de calor de la ciudad de Guayaquil corresponde a $+5,32^{\circ} \mathrm{C}$, valor que es tan fuerte como en las grandes ciudades (Shahmohamadi, Che-Ani, Ramly, Maulud, \& Mohd-Nor, 2010).

Al someter las variables a estudio de correlaciones se encontró que existe una relación de dependencia inversa de $\Delta \mathrm{T}$ con $\mathrm{SVF}$, así como una directa de $\Delta \mathrm{T}$ con h/w. La tabla3 muestra los resultados de este cálculo, para el que se estableció como criterio cualitativo que todo valor mayor a $+0,50$ y menor a $-0,50$ sería relevante, en consecuencia, las variables geométricas influyen significativamente sobre la temperatura del sector.

Tabla 3.

Estudio de correlación de variables.

\begin{tabular}{cccc}
\hline Variables & $\Delta \mathrm{T}[\mathrm{K}]$ & $\mathrm{SVF}[-]$ & $\mathrm{h} / \mathrm{w}[-]$ \\
\hline$\Delta \mathrm{T}[\mathrm{K}]$ & 1,00000 & & \\
$\mathrm{SVF}[-]$ & $-0,74259$ & 1,00000 & \\
$\mathrm{~h} / \mathrm{w}[-]$ & 0,67719 & $-0,88378$ & 1,00000 \\
\hline
\end{tabular}

A partir lo anterior se formuló el modelo de regresión lineal múltiple que describe el comportamiento de la variable $\Delta \mathrm{T}$ en función de las variables geométricas SVF y $\mathrm{h} / \mathrm{w}$ en la isla de calor del casco comercial de la ciudad de Guayaquil; la expresión numérica quedó como sigue:

$$
\Delta \mathbf{T}=6,302860-3,653178 \mathbf{S V F}+0,119063 \mathbf{h} / \mathbf{w}
$$

Esta ecuación bien podría emplearse para 'predecir' la temperatura en cada intersección vial del transecto estudiado tan sólo con sumar cualquier temperatura instantánea reportada por la estación fija 842030 SEGU al valor $\Delta \mathrm{T}$ proyectado, esto es posible debido a que todos los valores SVF y $\mathrm{h} / \mathrm{w}$ se comportan como características exclusivas e intrasferibles de cada intersección vial. No obstante, esta fórmula de regresión por sí sola no es capaz de modelar el impacto al confort higro-térmico que implica, para esto es necesario contar con más información meteorológica para introducirla en un diagrama bioclimático.

La estación fija 842030 SEGU en los días y rango horario de muestreo registró valores promedio para: velocidad del viento $=18,3 \mathrm{~km} / \mathrm{h}$, humedad relativa $=$ $68,34 \%$ y temperatura seca $=23,65^{\circ} \mathrm{C} . \mathrm{Si}$ se suma el incremento $\Delta \mathrm{T}=+5,32^{\circ} \mathrm{C}$ del punto05 a la última variable mencionada, se encuentra que la temperatura tentativa en este punto corresponde a $28,97^{\circ} \mathrm{C}$. Al ingresar el nuevo valor de temperatura y el de humedad relativa como par ordenado en la figura 7 es posible confirmar que el punto05 no está dentro de la zona de confort higro-térmico y que se requiere una velocidad del aire de $8.1 \mathrm{~km} / \mathrm{h}$ para lograrlo, no obstante, al tomar en cuenta la variable de velocidad del viento, se verifica que el valor reportado por la estación fija sobrepasa la medida correctiva. Teóricamente, mientras permanezca constante esa intensidad de ventilación, el punto05 presentará una sensación térmica tolerable para los peatones. 


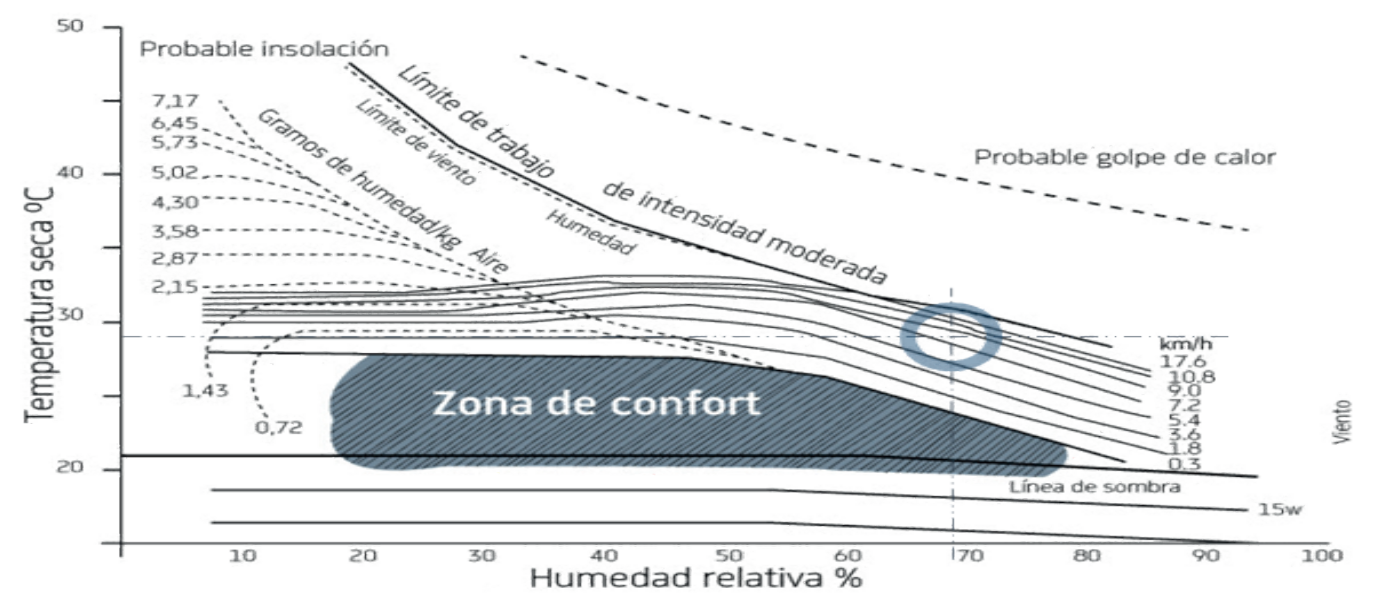

Figura 7. Carta bioclimática de Olgyay modificada para estudiar las condiciones de confort higro-térmico en áreas exteriores. ${ }^{8 * 9}$ El círculo en azul indica el lugar de ubicación de las coordenadas $\left(68.34 \% ; 28.97^{\circ} \mathrm{C}\right)$.

Para verificar la influencia de la geometría urbana sobre la forma de la capa del palio urbano bastó con pasar sobre el boulevard 9 de octubre un plano secante alineado con los puntos extremos del transecto de estudio, pues la unión sucesiva de sus veinte y tres intersecciones viales generó una línea poligonal abierta bastante aproximada a la recta imaginaria que une los puntos 01 y 23 (línea roja de trazo y doble punto en la figura 3). En la figura 8 se aprecia una línea roja de segmentos que es el límite de la capa del palio urbano y resulta de la unión de los puntos más altos de las cubiertas de los edificios; esto confirma que hay una intensificación del uso y ocupación del suelo a medida que los puntos de muestra se aproximan al punto 01 . El punto 05 está en esta densa zona y es el más caluroso.

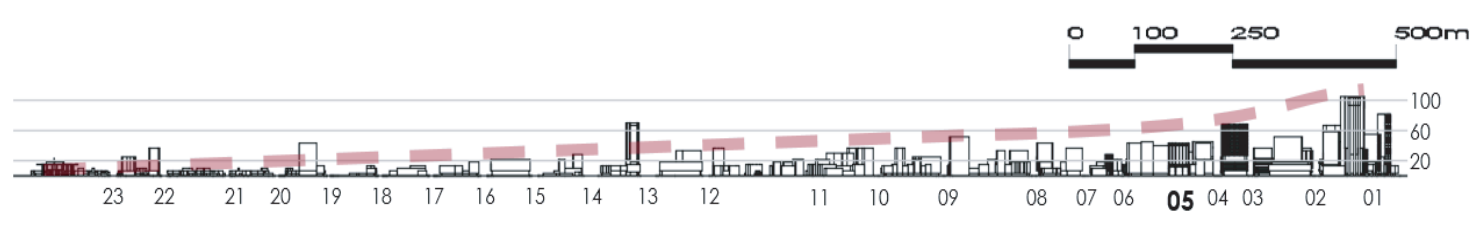

Figura 8. Sección longitudinal del transecto de estudio con vista hacia el norte. En el eje de las abscisas se indican las intersecciones viales y, en el de las ordenadas, la escala en metros para referenciar las alturas.

${ }^{8 *}$ La modificación de este diagrama consistió en descartar la información que se encuentra por debajo del número 20 en el eje de las ordenadas, debido a que los correctivos correspondientes no aplican para las temperaturas reportadas por correctivos correspondientes no aplican para las temperaturas reportadas por
estación $842030 \mathrm{SEGU}$, que en todos los casos fueron mayores o iguales a $21^{\circ} \mathrm{C}$.

${ }^{9}$ Fuente: http://www.mundohvacr.com.mx/mundo/wp-content/uploads/2013/10/ DIAGRAMA-bioclimatico-de-olgyay.jpg 
Una forma estadística de ver los mismos resultados se muestra en la figura 9, en donde la altura promedio de edificios de cada intersección vial está relacionada con la trayectoria del transecto desde 01 a 23; entiéndase como trayectoria de longitud $0,00 \mathrm{~m}$ la que le corresponde al punto $01 \mathrm{y}$ trayectoria de longitud 1923,12m la del punto 23 .

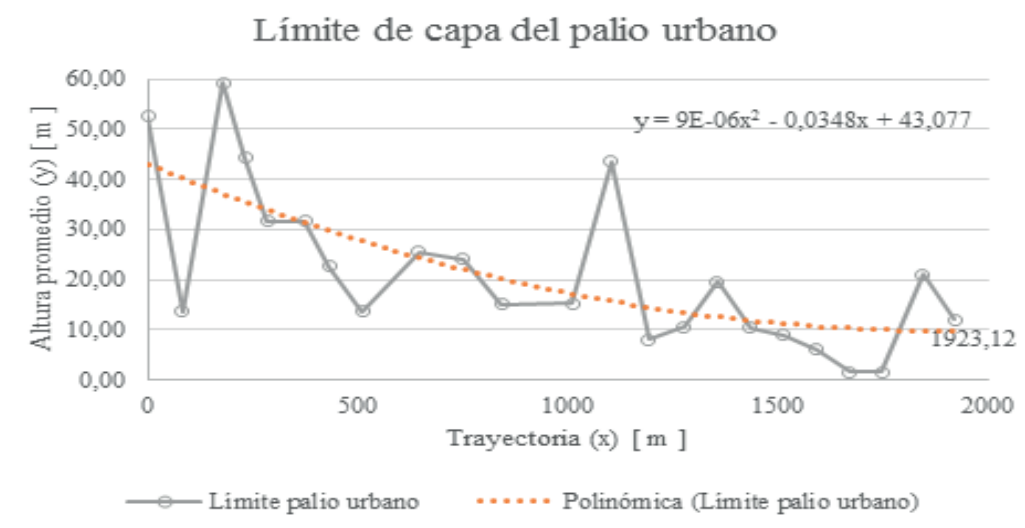

Figura 9. Representación estadística del límite de la capa del palio urbano. La línea segmentada en color anaranjado es la tendencia en función cuadrática que se ajusta al desarrollo de las alturas en función de la trayectoria de los puntos previamente tomada del modelo tridimensional.

La figura 9 muestra que el límite de la capa del palio urbano se caracteriza porque dieciséis de sus veintitrés puntos registran alturas promedio de edificios menores a $25,00 \mathrm{~m}$ y, al comparar los valores hasta ahora encontrados para las variables geométricas con los rangos correspondien- tes a dos posibilidades habituales de casco comercial para las ciudades en general, es factible calificar según la tabla4 al casco comercial-empresarial de la ciudad de Guayaquil como una zona urbana de edificios de mediana altura congregados de manera compacta (Stewart, 2011).

Tabla 4.

Comparación de rangos de variables geométricas determinantes del tipo de zona climática local.

\begin{tabular}{cccc}
\hline $\begin{array}{c}\text { Variables de } \\
\text { comparación }\end{array}$ & $\begin{array}{c}\text { Casco comercial de } \\
\text { Guayaquil }\end{array}$ & $\begin{array}{c}\text { Disposición compacta de } \\
\text { edificios de gran altura }\end{array}$ & $\begin{array}{c}\text { Disposición compacta de } \\
\text { edificios de mediana } \\
\text { altura }\end{array}$ \\
\hline SVF promedio & 0,63 & $0,20-0,40$ & $0,30-0,60$ \\
h/w promedio & 0,93 & $>2,00$ & $0,75-2,00$ \\
$\begin{array}{c}\text { Altura promedio de } \\
\text { edificios }\end{array}$ & 22,06 & $>25,00 \mathrm{~m}$ & $10,00-25,00 \mathrm{~m}$ \\
Ilustración & 0 & & \\
\hline
\end{tabular}

Nota: Los valores e imágenes de las dos columnas de la derecha fueron tomados y traducidos de la tesis "Redefining the urban heat island" por Iain Douglas Stewart, 2011, p.195. 
Por último, en cuanto a grandes ciudades, la tabla 5 confirma que el rango SVF del centro de Guayaquil se encuentra dentro de los reportados para grandes ciudades.

Tabla 5.

Comparación de rangos SVF típicos de la zona céntrica de grandes ciudades en varias localidades.

\begin{tabular}{lc}
\hline $\begin{array}{l}\text { Ubicación de zonas empresariales- } \\
\text { comerciales }\end{array}$ & Rango SVF \\
\hline Europa & $0,40-0,75$ \\
América del Norte & $0,28-0,86$ \\
Japón & $0,40-0,85$ \\
Japón & $0,26-0,78$ \\
Corea del Sur & $0,36-0,84$ \\
Guayaquil & $0,36-0,81$ \\
\hline
\end{tabular}

Nota: La tabla fue traducida y adaptada a partir de la información disponible en el reporte "Exploring the Urban Heat Island Intensity of Dutch cities" por L.W.A. van Hove, et al., 2011, p.27.

\section{Comentarios Finales}

En toda zona comercial-empresarial, cada metro cuadrado ocupado en el suelo abarca varios niveles de edificios en altura y denota una gran contribución de calor antropogénico debido al uso frecuente de equipos de climatización, la congestión vehicular y las propias actividades de la densidad poblacional flotante; a lo mencionado se suma la ganancia térmica de las coberturas del suelo y la descomunal capacidad calorífica de los edificios que reciben la energía del sol por radiación directa y difusa; sin olvidar que geométricamente las envolventes verticales también concentran calor en su superficie por reflexiones múltiples (Ooststroom, 2011).

El análisis geométrico del transecto estudiado demostró que donde hay más edificios altos congregados no hay suficiente cielo visible para que se produzca un intercambio térmico eficiente entre las capas adyacentes al límite del palio urbano a diferencia de donde están los de menor altura, así también lo confirma el registro de temperaturas representado en la figura6. Por otro lado, la figura3 sugiere que todo el sector denota un trazado urbano oblicuo en relación a la dirección suroeste del viento prevaleciente $\mathrm{y}$ redefine sus cañones urbanos como rutas ventiladas (Tatsuo) con potencial refrigerante para la ciudad cuando corre viento local desde el río Guayas hacia el interior del casco comercial y viceversa; sin embargo es necesario aclarar que para el cálculo del modelo de regresión no se incluyó la variable "velocidad del viento" debido a que en Guayaquil la ventilación es poco intensa y con escasas ráfagas durante la temporada de lluvias, lo que hace que el aire del cañón urbano quede 'atrapado' dentro de la capa del palio urbano y recepte los flujos de calor antropogénico y de 
la radiación liberada por las edificaciones $\mathrm{y}$ vías. El impacto ambiental queda en evidencia pues, al aumentar la temperatura disminuye el confort higro-térmico y se vuelve necesario acudir a sistemas activos de climatización que comprometen la calidad del aire; estos mecanismos extraen el calor de los espacios interiores y lo bombean hacia el exterior, de manera que se aporta más calor al aire del palio urbano que eventualmente será transferido a las superficies por convección.

De cualquier manera, quedó demostrado que el fenómeno de la isla urbana de calor existe en Guayaquil con una magnitud que se identifica con la de las ciudades de más de un millón de habitantes (Oke, 1997) y que responde de acuerdo a la intensidad aproximada de $22^{\circ} \mathrm{F}$ $\left(5,56^{\circ} \mathrm{C}\right)$ esperada en horas de la noche en ciudades californianas (CalEPA, 2017); también se encontró que sus parámetros geométricos tienen proximidad con los que se verifican en los cascos comerciales-empresariales de ciudades europeas, norteamericanas, japonesas y surcoreanas (van Hove, y otros, 2011), por tanto, su intensidad es mayor en los lugares donde la forma urbana está definida por una mayor densidad de uso y ocupación del suelo. El presente análisis comprobó que el patrón de respuesta térmica del suelo urbano de esta zona comercial-empresarial repite el patrón esperado dadas sus condiciones geométricas, además de que logró determinarse el impacto térmico y la forma espacial de la isla de calor de Guayaquil; razones que validan su prevención en zonas afines a contemplarse en las áreas de expansión de la ciudad mediante la planificación de trazados urbanos oblicuos respecto de la dirección del viento predominante (Chirag \& Ramachandraiah, 2011), con edificaciones no adosadas que permitan los flujos de aire en retiros y soportales al pie de vías cuya relación $\mathrm{H} / \mathrm{W}$ esté regulada con valores menores a 0,70 para evitar la formación de "situaciones de cañón" (Georgakis \& Santamouris, 2005).

\section{Referencias}

CalEPA. (2017). Understanding the Urban Heat Island Index. Retrieved from California Environmental Protection Agency: https://www.calepa.ca.gov/climate / u rban-heat-is land-index-for-california/understanding- $t$ he-urban-heat-island-index/

Chirag, D., \& Ramachandraiah, A. (2011). A simple technique to classify urban locations with respect to. (Q. Chen, Ed.) Building and environment, 46, 1321-1328. Retrieved from http://www.academia.edu/19845811/A_simple_technique $\mathrm{to}_{-} \mathrm{class}$ i fy_urban_locations_with_respect to_human_thermal_comfort_Pro posing_the_HXG_scale

Georgakis, C., \& Santamouris, M. (2005, may). Canyon effects: Calculation of wind speed in an urban street canyon with the aid of a semi-empirical model based on experimental data. In M. Santamouris (Ed.), 1st International Conference on Passive and Low Energy Cooling for the Built Environment. Santori- 
ni, Greece: Heliotopos conferences. Retrieved from http://www.inive.org/

Gonçalves Dos Santos, I., Gazzola De Lima, H., \& Sad De Assis, E. (2015). A comprehensive approach of the sky view factor and building mass in an urban area of the city of belo horizonte Brazil. (T. F. Gerais, Ed.) Retrieved from https://www.researchgate.net/publication/264840907

Moreno García, M. (1997). Revistas Investigaciones Geográficas - \#17 (1997). Una propuesta de terminología castellana en climatología urbana. (I. Universidad de Alicante, Ed.) doi:10.14198/INGEO1997.17.08

Oke, T. R. (1997). Urban Climates and Global Environmental Change. In R. Thompson, \& A. H. Perry, Applied climatology: principles and practice. (pp. 273 - 287). London, New York. Retrieved from http://trove.nla.gov.au/wor$\mathrm{k} / 15822391$ ? selectedver sion $=\mathrm{NBD} 12779982$

Ooststroom, H. v. (2011, August 30).

Urban Heat Island adaptation through Urban Planning and Design: the struggle of the city of Los Angeles. Los Angeles: University if California, Irvine. Retrieved from http://www-sre.wu-wien.ac.at/neurus/van_Ooststroom.pdf
Rajagopalan, P., Chuan Lim, K., \& Jamei, E. (2014). Urban heat island and wind flow characteristics of a tropical city. (D. U. School of architecture and Built Environment, Ed.) Solar Energy, 107, 159-170. doi:https://doi.org/10.1016/j.solener.2014.05.042

Shahmohamadi, P., Che-Ani, A., Ramly, A., Maulud, k., \& Mohd-Nor, M. (2010, june 30). Reducing urban heat island effects: A systematic review to achieve energy consumption balance. International Journal of Physical Sciences 5 , no. 6 (2010): 626-636. Retrieved from http://academicjournals.org/journal/IJPS/article-abstract/DBB8AC426195

Stewart, I. D. (2011). Redefining the urban heat island. Local climate zone classification systems, 368. Vancouver, Canada: University of british Columbia. doi:10.14288/1.0072360

Takkanon, P. (2016). UHI and Thermal Performance of Office Buildings in Bangkok. (L. Ding, F. Fiorito, \& P. Osmond, Eds.) Procedia Engineering, 180, 1784. doi:https://d o i . o r g/10.1016/j.proeng.2017.04.183

Tatsuo, A. (n.d.). Creating the 'Wind Paths' in the City to Mitigate Urban Heat Island Effects. A Case Study in Central District of Tokyo. Retrieved from http://www.kenken.go.jp: 
